
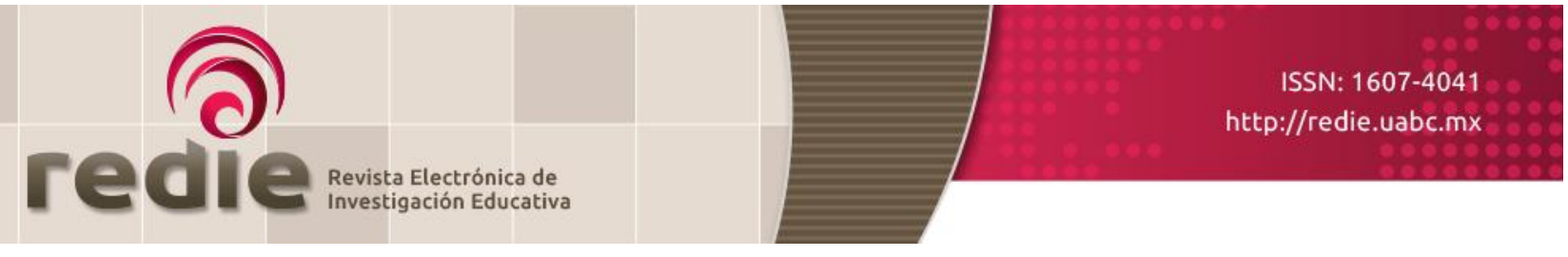

Vol. 19, Núm. 3, 2017

\title{
Pautas para la aplicación de métodos de enseñanza musical desde un enfoque constructivista
}

\author{
Guidelines for Implementation of Music Teaching Methods \\ from a Constructivist Approach
}

José Luis Navarro Solís (*) jolunasmx@yahoo.com.mx

(*) Centro Nacional de Investigación, Documentación e Información Musical “Carlos Chávez"

(Recibido: 16 de marzo de 2014; Aceptado para su publicación: 31 de marzo de 2016)

Cómo citar: Navarro, J. L. (2017). Pautas para la aplicación de métodos de enseñanza musical desde un enfoque constructivista. Revista Electrónica de Investigación Educativa, 19(3), 143-160. https://doi.org/10.24320/redie.2017.19.3.675

\begin{abstract}
Resumen
Muchos músicos se han dedicado a dar clases, lo que dio origen a la educación musical; sin embargo, en varias escuelas de música la formación pedagógica dada a los escolares es elemental o nula y se va aprendiendo por medio del método ensayo-error. Parte del problema con la didáctica es la utilización de un método de enseñanza, pues no existe una correlación demostrable entre el docente y el éxito que pueda tener un método en cada uno de los contextos. Este artículo ofrece una revisión teórica de conceptos pedagógicos de autores representativos, seguidos de una serie de recomendaciones para desarrollar o implementar métodos de enseñanza musical en diversos entornos educativos con el apoyo de algunas teorías derivadas del constructivismo.
\end{abstract}

Palabras clave: Educación musical, métodos de enseñanza, constructivismo.

\section{Abstract}

Many musicians have made a living by teaching, which gave rise to music education; however, in many music schools, the teacher training given to students is basic or non-existent, and they learn by trial and егror. Part of the problem with teaching is the use of a teaching method, as no provable correlation exists between teachers and the success rate of a given method in each context. This paper offers a theoretical review of pedagogical concepts from representative authors, followed by a series of recommendations for introducing or developing music teaching methods in various learning environments, supported by theories derived from constructivism. 


\section{Introducción}

Quizá la poca conexión entre teoría y práctica en la enseñanza artística generó la idea de que la música es sólo para personas con un talento especial (Piñeiro, 1986), aunque es posible que la falta de métodos adecuados aumente este problema. Este trabajo conecta conceptos de la teoría constructivista con algunos modelos pedagógico-musicales, con el objetivo de ofrecer un compendio que auxilie a profesores de música, principalmente a aquellos que se inician en la actividad docente. A continuación se presentan las teorías implementadas y su adaptación para el despliegue de estrategias para la enseñanza musical.

El constructivismo propone que el conocimiento es una construcción del individuo y se efectúa desde sus esquemas previos, por ello es muy importante la habilidad del docente para diagnosticar los conocimientos previos de los estudiantes (Lara, 2005). El constructivismo social agrega que el aprendizaje se desarrolla por medio de la interacción, y el desarrollo del lenguaje es fundamental para el incremento de las habilidades cognitivas (Vygotsky, 2008). Además, algunos autores opinan que la música presenta etapas evolutivas similares al lenguaje, pues precisa de una impregnación anterior a la práctica, llega a la conciencia con la imitación, la repetición y la asimilación (Muñoz, 2003; Gardner, 2005). En este apartado se recuperan los conceptos principales de las teorías que reflejan los supuestos expuestos.

\subsection{Teoría del desarrollo del proceso de aprendizaje en el niño}

Jean Piaget realizó muchas exploraciones para indagar cómo aprende el ser humano. A continuación, se enlistan algunas investigaciones de este autor utilizables para la enseñanza musical.

De acuerdo con esta teoría, la infancia dura más cuanto superior es la especie, y es necesaria para el aprendizaje de los seres vivos. Piaget (1996) propone las siguientes etapas de desarrollo:

a) Inteligencia sensorio-motriz (antes de los 18 meses). Precede al lenguaje. Se construyen la noción de objeto, espacio, tiempo y causalidad, que posteriormente construirán el pensamiento. Los niños usan sus capacidades sensomotoras para explorar su medio ambiente.

b) Representación preoperatoria (2 a 7-8 años). Comienza con el lenguaje. El niño tendrá la noción de un espacio general que engloba lo bucal, lo visual, lo táctil y lo auditivo. Empieza a usar símbolos, responde a objetos y eventos de acuerdo a lo que parecen. Existe el juego que se convertirá en juego simbólico.

c) Operaciones concretas (7-12 años). El infante desarrolla pensamiento lógico que no versa sobre enunciados verbales y que se aplica únicamente sobre los propios objetos manipulables, es capaz de coordinar operaciones en el sentido de la reversibilidad.

d) Operaciones proposicionales o formales (12 años en adelante). Desarrollan el pensamiento sistemático y abstracto que llega a un nivel de equilibrio alrededor de los 15 años. Son capaces de desarrollar procesos lógicos de razonamiento deductivo sobre una hipótesis y proposiciones.

Estas etapas siguen siempre este orden, aunque las edades pueden cambiar de un contexto a otro. Asimismo, el aspecto psicosocial es fundamental para el desarrollo de conceptos y esquemas representativos ligados a la expresión verbal del niño (Piaget, 2006). Pero el desarrollo psicosocial se subordina al espontáneo y psicológico (Piaget, 1996). Las variaciones en la duración se dan a razón de los siguientes factores:

a) La herencia-maduración interna. Es insuficiente porque jamás juega en estado puro. Interviene un efecto de maduración indisociable del aprendizaje o la experiencia.

b) La experiencia física. Constituye un factor esencial, pues la lógica del niño proviene de las acciones que ejerce sobre los objetos. 
c) La transmisión social. Para que se establezca una transmisión entre el medio social y el medio educado es preciso que exista una asimilación por parte del niño.

d) La equilibración. Es la compensación por reacción del sujeto a las perturbaciones exteriores que lleva a la reversibilidad operatoria.

La formación del símbolo en el niño. La representación simbólica se refiere a la posibilidad de personificar una cosa por medio de otra, es decir, un sistema de signos sociales por oposición a los individuales, para lograr este proceso el niño debe poder imitar el modelo ausente. La imitación lleva a la representación cognoscitiva por medio del proceso:

a) Imitación. El niño imita sonidos y movimientos que pueden alcanzar el nivel de actividades lúdicas y conductas de forma innata. Es indispensable el interés.

b) Juego. Es constituido como un nivel superior de imitación que procede por relajación del esfuerzo adaptativo por el puro placer de dominar las actividades.

c) Imaginación. Los deseos frecuentemente inconfesables son canalizados. Este mecanismo indica que el niño se ha sociabilizado, se apoya del sueño que prolonga el juego simbólico.

d) Pensamiento o representación cognoscitiva. El juego se vuelve simbólico y se prolonga bajo simbolismo inconsciente. Prepara la construcción representativa, la asimilación libre se reduce a la imaginación creadora, la palabra funciona como signo.

Desarrollo de la noción del tiempo en el niño. Para Piaget (2005) hay dos formas de distinguir el tiempo: la operativa, que consiste en relaciones de sucesión y duración sobre las operaciones análogas y lógicas; y la intuitiva, que son las relaciones de sucesión y duración dadas en la percepción inmediata.

El tiempo está ligado a los recuerdos, a un proceso causal complejo o a movimientos bien delimitados que requieren, para que el infante lo perciba, el despliegue de los siguientes estadios: 1) el niño no consigue construir un tiempo único, pues carece de reversibilidad en relación a los sucesos; 2) Aunque el niño invierte la relación del tiempo y de la velocidad, puede llegar a igualar las duraciones sincrónicas sin lograr articular correctamente las duraciones parciales, y 3) el niño llega a la consecución de un tiempo operativo, cualitativo y métrico.

\subsection{Teoría del desarrollo del pensamiento}

Para Vygotsky (2008), el lenguaje es la base material del pensamiento, pues en el significado se encuentra la relación entre la inteligencia y la palabra. El lenguaje del adulto y del infante es distinto, pues las formas superiores del intercambio humano son viables si el pensamiento del adulto refleja una realidad a través de los conceptos y no pueden ser transmitidos a los niños; por esta razón, para que el proceso ocurra debe surgir un problema que pueda solucionarse a través de la formación de nuevos conceptos. Vygotsky considera las siguientes etapas o estadios del desarrollo de la inteligencia:

1. Etapa primitiva o natural. Corresponde al lenguaje pre-intelectual y al pensamiento pre-verbal.

2. Etapa de la psicología simple. Definida por el desarrollo del lenguaje. El niño experimenta con las propiedades físicas de su propio cuerpo y de los objetos a su alrededor.

3. Etapa egocéntrica. Puede distinguirse por el manejo de signos y operaciones externas que son utilizadas por los infantes como ayuda en la solución de problemas internos.

4. Etapa de crecimiento interno. La operación externa se convierte en interna. El niño comienza a operar con relaciones inherentes y signos interiorizados. 
Constructivismo social. La zona de desarrollo próximo es el trayecto entre el nivel real de despliegue establecido por la habilidad de solucionar autónomamente un problema y el nivel de desarrollo potencial acordado por medio de la solución de un problema, con la guía de un adulto o de otro compañero. Los niños, por medio de la acción, reconstruyen su mundo sociocultural a la vez que se socializan y forman sus funciones psicológicas superiores y su conciencia (Hernández, 2010). El aprendizaje se entiende como un proceso de cooperación por medio del cual los sujetos interactúan e intercambian distintos recursos cognitivos y estructuras interpretativas de la realidad y predominan las que, por medio del consenso social, se imponen como las más adecuadas (Santoianni y Striano, 2006). Otro concepto derivado es el del aprendizaje colaborativo, que se fundamenta en el principio educativo de que para un estudiante el mejor profesor es otro estudiante (Ferreiro, 2007). Vygotsky (1979) afirma que los procesos de enseñanza que se llevan a cabo de manera grupal tienen la ventaja de la interacción entre iguales.

Conceptos didácticos desde la perspectiva de Ausubel. Para Ausubel, Novak y Hanesian (2006) hay dos formas en se puede aprender: una que se refiere a la manera en que el escolar adquiere los nuevos conceptos, y otra que es la forma en que los nuevos conceptos son introducidos a la estructura cognitiva. Con este fundamento proponen un concepto y una herramienta didáctica:

1. Aprendizaje significativo. Es la forma natural en que se aprende. La ganancia de significados necesita material potencialmente significativo y actitud de aprendizaje significativo. Los procesos psicológicos involucrados esperan una estructura cognitiva pre-existente en el sujeto que asimilará los nuevos conceptos. Dicha asimilación pasa en función de las relaciones jerárquicas que el educando establece entre los conceptos en los que el conocimiento más inclusivo asimila otros más específicos, de manera que los conocimientos adquieren un nuevo significado.

2. Mapas conceptuales. Pueden utilizarse para la elaboración de procesos curriculares y el diseño de módulos de instrucción lógicos y potencialmente significativos. También son útiles para que los materiales didácticos puedan ser conceptualmente más transparentes; son instrumentos válidos para crear y compartir contenidos de referencia entre profesor-alumnado e incluso entre estudiante-estudiante.

\subsection{Teoría de formación del pensamiento musical de Moog}

El desarrollo de esta teoría se plantea en paralelo con la epistemología genética piagetiana, aunque propone un período de estimulación temprana necesario para el desarrollo de las capacidades musicales, en el que es importante la exposición de los infantes al baby talk, que se caracteriza por tener connotaciones musicales y lingüísticas precursoras al desarrollo del lenguaje, así como a las canciones de cuna, pues además de exponerlo a elementos musicales, capacitan al bebé para percibir modulaciones de voz y una carga emocional musical (Moog, 1976). A continuación se enumeran las etapas de desarrollo:

Desarrollo del canto espontáneo. Se apertura con el predominio de intervalos descendentes y canciones breves con repeticiones de palabras de un tono sin valor rítmico con pausas para respirar. Hacia los 2 años los infantes pueden reproducir canciones más largas y organizadas e intervalos de segunda y tercera. A los 4 años pueden crear canciones popurrís procedentes de otras conocidas aunque alteran palabras y ritmo. Hacia los 5 años disminuye la frecuencia de canciones espontáneas, pues se presenta por primera vez la preocupación de la precisión.

Desarrollo de la imitación de canciones. Inicia con la repetición de algunos fragmentos de letras, así como patrones rítmicos y tonales hasta llegar a desplegar el contorno melódico y rítmico. De los 3 a 4 años pueden repetir una canción completa, aunque sostienen el ritmo, melodía o tonalidad con mucha dificultad. A los 5 años pueden reproducir canciones infantiles con precisión, así como alcanzar una extensión de décima con desplazamientos de intervalos máximos de sexta. De los 6 a los 7 años los infantes toman conciencia de la naturaleza del intervalo y la duración del sonido, se asimila la jerarquía del sistema melódico y pueden concluir las frases en grados tonales; la extensión alcanzada es de doceava y pueden cantar intervalos máximos de octava. Se considera que a los 8 años es la mejor edad de la voz, pues alcanza una extensión de catorceava y, con previa formación, pueden cantar a cuatro voces. 
Desarrollo de la habilidad rítmica. En los primeros años de vida predomina el balanceo y los movimientos ondulares, aparecen signos tempranos de coordinación musical y hacia los 2 años y medio pueden imitar y crear actividades rítmicas. A partir de los 3 años es conveniente trabajar la lateralidad, aunque no hay adelantos hasta los 5 . En esta edad se desarrolla más el juego imaginativo y el niño prefiere sentarse a escuchar y se desarrolla la sincronización de movimientos con la música. Hacia los 6 años muestran afición por estructuras rítmicas regulares, así como capacidad de sincronizar extremidades inferiores y superiores, pero dificultad para mantener el pulso. A los 7 años la coordinación puede ser perfecta. A los 8 años se presenta el desarrollo de capacidades motrices y de la expresión corporal, así como la simbolización y abstracción del ritmo necesaria para comprender la métrica musical, la escritura rítmica y los cambios de compás. A los 9 años se puede desarrollar polirritmia.

Desarrollo de la capacidad melódica. Hay controversia si las primeras manifestaciones musicales son melódicas o rítmicas. El balbuceo es precursor del habla y aparece de los 2 a los 8 meses posiblemente como respuesta a la música oída, se realiza sobre una vocal o pocas sílabas y con un ritmo pobre. La discriminación de alturas va progresando de los 7 a los 14 años.

Desarrollo de la adquisición de la tonalidad. A los 5 años los niños pueden detectar cambios de tonalidad, a los 6 se tiene escasa comprensión de las cadencias en las estructuras tonales. A los 7 años se detectan cambios de tonalidad en melodías y a los 8 cambios del modo mayor cuando mejora la adquisición de la tonalidad y se reconoce la diferencia entre tónica, dominante y cadencia perfecta. A los 9 años pueden seleccionar la tónica. A los 10 perciben las estructuras rítmicas, melódicas y armónicas.

Aspectos psicomotrices en la interpretación musical. La formación de conceptos musicales requiere del despliegue de operaciones cognitivas, así como del desarrollo de la atención y la memoria. Estas operaciones son requeridas para la interpretación musical independiente al instrumento o tipo de música de que se trate (Furnó, 2005):

Capacidades físicas. Representan las particularidades psicológicas de los individuos que son distintas de un sujeto a otro, por lo que un individuo puede capacitarse mejor y más rápido. Pero las capacidades no son innatas, lo son las disposiciones o aptitudes que son parte de las particularidades físico-anatómicas de todo individuo. Las capacidades básicas respecto al desarrollo musical están ligadas con el oído, el tacto, la vista y el sistema nervioso.

Habilidades. Significa poder utilizar favorablemente los datos, conocimientos y conceptos logrados a fin del esclarecimiento de las propiedades sustanciales de los objetos y la solución de teorías. Este aspecto se ejercita con participación mayor de la conciencia.

Hábitos. Se basa en un sistema que puede excitarse a voluntad para volver a realizar la actividad por la que fue realizada. Se entiende que una habilidad se alcanza cuando se ha logrado un sistema permanente a través de la práctica.

Aspectos psicológicos. En los años previos a la adolescencia el aprendizaje musical puede ser más viable a través de la imitación. En la adolescencia inician algunos procesos de cambio y los individuos gradualmente estarán aptos para sintetizar el mundo a través del desarrollo del pensamiento abstracto.

Atención. Está relacionado con procesos de reconocer, imaginar, recordar y pensar. Es una propiedad de la percepción caracterizada por su naturaleza selectiva que permite extirpar sólo la zona de información a ser procesada. Define dos tipos: la atención selectiva que consiste en la atención máxima de concentración sobre un objeto y con resistencia a otro estímulo; y la atención distribuida que es aquella que se reparte entre dos o más objetos de atención. Escuchar demanda el tipo de atención distribuida y la interpretación de un instrumento musical requiere de ambas.

Memoria. Este aspecto es fundamental para lograr la firmeza de los conocimientos adquiridos. Se clasifica en tres tipos: memoria a muy corto plazo denominada registro de información sensorial; memoria a corto plazo que procesa datos y mantiene resultados de operaciones de manera transitoria; y memoria a largo 
plazo que reconoce dos tipos de representación mental, una procedural que corresponde a la memoria de acción y de personas, y otra declarativa que corresponde a la memoria semántica y episódica. Piñeiro (1986) clasifica la memoria en dos tipos: la memoria mecánica en la que interviene un mínimo de razonamiento y auxilia a la mente a recordar cosas concretas como símbolos; y la memoria intelectiva que concierne a las relaciones entre sonidos, armonías y contenido musical. Hay que agregar que cada uno de los cinco sentidos tiene una memoria: vista, olfato, gusto, tacto y audición.

\section{Elementos generales de la pedagogía: modelos de enseñanza musical}

Un modelo se integra de dos elementos: un contenido (lo que se va a enseñar) y una forma de implementación (cómo se va a enseñar). En correspondencia con Bunge (1980) método es un procedimiento material o conceptual regular, explícito y repetible por medio del cual es posible conseguir un propósito. A continuación se describen los principales modelos pedagógicos, ciertos sistemas didácticos y algunas metodologías de enseñanza musical.

\subsection{Modelos pedagógicos}

Un modelo de enseñanza es el retrato de un contexto educativo, aunque hay más connotaciones sobre el término que van desde la organización de currículos, cursos o unidades didácticas (Joyce, Weil y Calhoun, 2006). En la tabla I se presenta una clasificación con base en las nociones que comparten sobre la forma en que aprenden los individuos. Los modelos presentan tres elementos en común: un fundamento teórico; una sistematización de implementación didáctica y adaptabilidad en torno a su aplicación a diversos estilos de aprendizaje; y requerimientos de diversas asignaturas.

Tabla I. Modelos pedagógicos (Joyce et al., 2006)

\begin{tabular}{lll}
\hline Fundamento & Modelo & Teóricos \\
\hline \multirow{4}{*}{ Modelos Sociales } & Interdependencia positiva & Johnson, Johnson, Calderon, Cohen \\
& Indagación estructurada & Slavin \\
& Investigación grupal & Dewey, Thelen \\
& Juego de roles & Shaftel \\
Indagación jurisprudencial & Oliver, Shaver \\
Modelos de diseño & Teoría de los sistemas conceptuales & Hunt, Harvey, Schroeder \\
& Desarrollo cognitivo & Piaget \\
Modelos de & Condiciones de aprendizaje & Gagné \\
procesamiento & Pensamiento inductivo & Taba \\
de la información & Formación de conceptos & Bruner \\
& Mnemotecnia (ayuda memoria) & Pressley, Levin, Anderson \\
& Organizadores previos & Ausubel \\
Indagación científica & Schwab \\
Modelos personales & Entrenamiento para la indagación & Suchman \\
& Sinéctica & Gordon \\
& Enseñanza no directiva & Rogers \\
& Elevar la autoestima & Maslow \\
Modelos de conducta & Instrucción directa & Bloom, Block \\
& Simulación & Good, Brophy, Gereiter, Englemann, Becker \\
& Aprendizaje social & Smith, Smith \\
\hline
\end{tabular}

\subsection{Sistemas didácticos}

Los sistemas didácticos se refieren a la estructura de una clase de cualquier disciplina, a continuación se describen brevemente:

Sistema didáctico de Savin. Cada clase debe ser la continuidad de la anterior y pueden presentarse eslabones a través de combinaciones que deben estar sujetas a reglas internas y fluir espontáneo. 


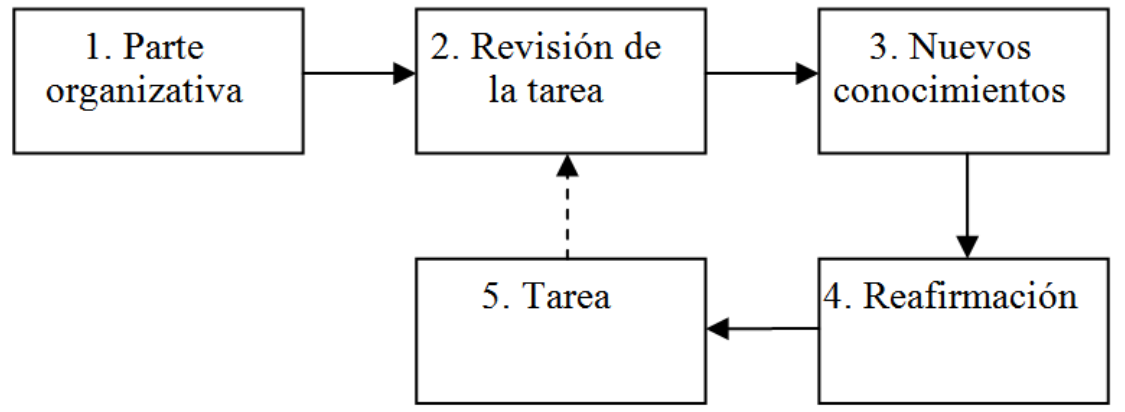

Figura 1. Estructura de la clase combinada según Savin (Piñeiro, 1986)

Sistema didáctico de Sierra. En este sistema se implementan las TIC en clase a fin de transmitir conocimientos y promover el uso cotidiano de la computadora. Primero se realiza un análisis de la sociedad para diseñar actividades congruentes con el conocimiento tecnológico de los estudiantes.

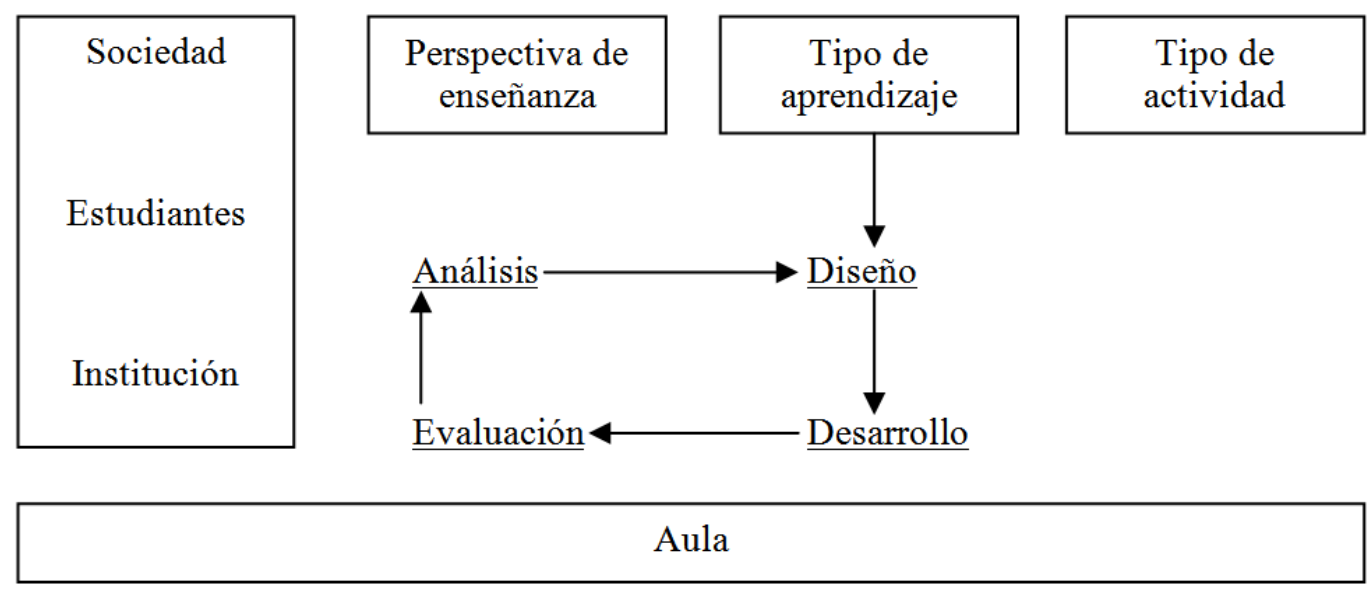

Figura 2. Proceso de diseño de actividades educativas con informática (Sierra, 2002)

Sistema didáctico de Burgos. El profesor expone ejemplos que después los pupilos repiten. Se requiere planear las actividades a través de metas a corto o largo plazo y establecer una línea progresiva con una secuencia entre los contenidos, para lo que propone diez objetivos: profundizar en las características técnico-interpretativas del instrumento; desarrollar la capacidad de auto-aprendizaje; desarrollar una técnica, personalidad y creatividad interpretativa; valorar y desarrollar el juicio crítico; desarrollar métodos y estrategias de estudio; conocer e interpretar el repertorio contemporáneo; desarrollar la capacidad de juicio estético; comprender y aplicar los principios básicos de anatomía funcional; desarrollar las cualidades técnico-interpretativas a partir de ejercicios de mecanicismo y técnica creados por el propio alumno; y valorar el análisis comprensivo de la música (Burgos, 2007).

\subsection{Métodos de enseñanza musical}

De acuerdo con Alsina (2007, p. 15) "[...] la metodología está definida como ciencia o estudio del método, o de los métodos, se nutre de modelos, conceptos y teorías, para luego desembocar en un esquema adecuado [...]." En los múltiples contextos donde se realizan procesos de enseñanza y aprendizaje se implementa algún método de enseñanza, aunque existen varias formas en que se implementan, desde el eclecticismo absoluto hasta el seguimiento paso a paso. Existe una amplia variedad de métodos aplicables a los distintos contextos de enseñanza: 
Métodos de enseñanza instrumental. Podemos definirlos como obras que enseñan los elementos necesarios para interpretar las obras escritas para instrumentos musicales, mismos que para cumplir sus propósitos tienen como contenido una serie de ejercicios, estudios, teorías, máximas, observaciones filosóficas y otros dictados de forma progresiva y de manera implícita o explícita. Su objetivo es el dominio de la lectoescritura que emplean y el desarrollo de la técnica instrumental. Su peculiaridad es que soportan su praxis musical con el apoyo de un instrumento, de hecho, existen métodos cuya finalidad es aprender a ejecutar un instrumento hasta llegar a su pleno dominio.

a) Métodos de enseñanza hasta el S. XIX. Se publicaron varios textos aplicables a la enseñanza desde compendios de estudios, textos con fórmulas para ejercitar la musculatura y métodos de instrucción. Algunos de estos textos siguen siendo utilizados.

b) Métodos de enseñanza de la primera mitad del S. XX. Algunos textos de este período surgieron a partir del intento de sistematizar los modelos heredados del siglo XIX. Unos incluyen tratados de organología, teoría de la música y diversos ejercicios para ejercitar las partes del cuerpo empleadas para ejecutar el instrumento.

c) Métodos de enseñanza de la segunda mitad del S. XX. A partir de 1950 se realizó una considerable publicación de métodos y aunque varios autores repitieron las fórmulas decimonónicas, otros tomaron en cuenta aspectos no tratados con anterioridad. Se distinguen las siguientes líneas:

- Métodos de enseñanza Infantiles. Desarrollan habilidades musicales en la medida de las posibilidades psicológicas e intelectuales de los infantes. Generalmente inician con melodías infantiles sencillas con acompañamiento para que el alumno toque con el profesor.

- Métodos de enseñanza especializados en aspectos técnicos. Este es un desarrollo de metodologías empleadas desde el siglo XIX. Los textos se enfocan en el desarrollo de un aspecto técnico en particular.

- Métodos de enseñanza de innovación pedagógica. Las principales novedades son la introducción del uso de colores y melodías populares.

- Métodos de enseñanza con apoyo tecnológico. Utilizan el soporte de una grabación en audio, video o alguna plataforma en Internet.

- Métodos para el desarrollo de técnicas extendidas. ${ }^{1}$ Se han desarrollado para la interpretación de la música contemporánea de vanguardia en la que emplean lenguajes con este tipo de técnicas.

Citar todos los métodos de enseñanza instrumental, aun realizando una selección de los más representativos, sería una tarea para otro trabajo de investigación; sin embargo, en la tabla II se hace mención de metodologías desarrolladas para diversos instrumentos musicales que han tenido auge en años recientes.

1 Se conocen las técnicas de interpretación utilizadas en música mediante las que se ejecutan técnicas no convencionales, ni tradicionales ni ortodoxas de cantar o tocar instrumentos musicales con el objetivo de obtener sonidos inusuales (Wikipedia, 2014). 
Tabla II. Métodos de enseñanza instrumental

\begin{tabular}{|c|c|}
\hline Método & Descripción \\
\hline Suzuki & $\begin{array}{l}\text { El proceso escuchar-repetir-memorizar es la forma natural con la que el cerebro funciona mejor, el } \\
\text { ambiente correcto se puede establecer en el hogar, la escuela o lecciones privadas. En una lección } \\
\text { el niño repite el material hasta que pueda realizarlo con éxito. La lectura y notación de música se } \\
\text { introducen una vez que el niño ha alcanzado un poco de habilidad técnica con el instrumento. }\end{array}$ \\
\hline Tort $^{2}$ & $\begin{array}{l}\text { Este método se desarrolla por medio de cuatro etapas. 1) Estimulación temprana (3 meses-1 año } \\
11 \text { meses): los niños comienzan a experimentar la música realizando, con alguno de sus padres y } \\
\text { la maestra, juegos musicales; } 2 \text { ) Maternal ( } 2-3 \text { años): El alumno se inicia en el canto, intercalando } \\
\text { en sus juegos musicales actividades en las cuales acompañan sus cantos con un grupo de pequeños } \\
\text { instrumentos de percusión; } 3 \text { ) Motivación Musical (3-5 años): Son } 3 \text { niveles que corresponden al } \\
\text { jardín de niños (en México) las actividades se dividen en Juegos abiertos, en donde continúan sus } \\
\text { actividades en las que el cuento, la pantomima y elementos teatrales se combinan con la música, } \\
\text { y Juegos cerrados, en los que la actividad de los niños va dando forma a un conjunto coral y a un } \\
\text { conjunto instrumental; y 4) Educación Musical por Nota (6-12 años): Abarca lo correspondiente } \\
\text { a la educación primaria. }\end{array}$ \\
\hline Yamaha & $\begin{array}{l}\text { Esboza su inicio entre los cinco años dependiendo del instrumento que es el eje del aprendizaje } \\
\text { musical. Tiene como finalidad escuchar, reconocer y tocar en el instrumento, además de trabajar el } \\
\text { acompañamiento, la improvisación y la ejecución solo y en pequeños ensambles. Una distinción } \\
\text { con los métodos Suzuki y Tort es que inicia con la lectoescritura (Jorquera, 2004) }\end{array}$ \\
\hline
\end{tabular}

Métodos para la enseñanza social de la música. Cuando se habla de métodos para la enseñanza social de la música se hace referencia a textos que abordan medios que el profesor facilitará al alumnado para que adquieran ciertos conceptos e interioricen una actitud. Estos se aproximan a la música desde edades tempranas con instrumentos musicales simples de percusión, la voz o el mismo cuerpo, sin el afán de formar músicos a nivel profesional, sino más bien de sensibilizar a los infantes hacia las cualidades de la música: el ritmo, los sonidos, etc. (Burgos, 2007). En la tabla III se enlistan algunos autores que han desarrollado métodos de enseñanza musical.

Tabla III. Métodos para la enseñanza social de la música

\begin{tabular}{|c|c|}
\hline Método & Descripción \\
\hline Bayard & $\begin{array}{l}\text { Tiene como finalidad ofrecer desde los } 6 \text { años una formación centrada en la } \\
\text { música clásica, popular y el canto gregoriano. }\end{array}$ \\
\hline Dalcroze & $\begin{array}{l}\text { Permite adquirir el sentido musical por medio del ritmo corporal, a través de esta } \\
\text { metodología se enseñan los diferentes parámetros de la música con relación al } \\
\text { movimiento y al espacio, en grupo y, en muchas ocasiones, con material auxiliar } \\
\text { favorece y valoriza la percepción sensorial. }\end{array}$ \\
\hline Kodàly & $\begin{array}{l}\text { Se basa en la educación del oído y en la adquisición de una voz bien educada para } \\
\text { el canto antes de introducir al alumno en la práctica de cualquier instrumento. }\end{array}$ \\
\hline Martenot & $\begin{array}{l}\text { Consiste en singularizar cada una de las materias de que se compone el lenguaje } \\
\text { musical y encontrar una respuesta global que conduzca a una ágil comprensión } \\
\text { de la lectura a primera vista que intenta ir más allá del descifrado y la idea } \\
\text { musical. }\end{array}$ \\
\hline Orff & $\begin{array}{l}\text { Se despliega a partir del interés del educando a través de la conjugación del } \\
\text { movimiento, la música y el lenguaje. Para ello, implanta instrumentos de } \\
\text { percusión relacionando los ritmos musicales con el lenguaje. Da importancia a la } \\
\text { improvisación y a la creatividad. }\end{array}$ \\
\hline Willems & $\begin{array}{l}\text { Se basa en que los niños tengan la experiencia directa con la música, además de } \\
\text { llevar un orden de desarrollo idéntico al de la adquisición de la lengua materna. }\end{array}$ \\
\hline
\end{tabular}

${ }^{2}$ Aunque la primera etapa de este método puede encajar en los métodos para la enseñanza social decidimos clasificarlos aquí, pues su finalidad primordial es acercar a los educandos a la ejecución con algún instrumento musical. Para más información el lector puede consultar el sitio http://artene.org.mx/inicio_artene.html 


\section{Elementos para la aplicación de métodos para la enseñanza musical}

En este apartado se presenta un recuento de los elementos más relevantes a considerar para la implementación de métodos de enseñanza musical. Iniciamos con nuestra postura sobre algunos conceptos de autores enmarcados en el constructivismo.

En la tabla IV se contrastan las aportaciones de Piaget (1962) y Vygotsky (1979) sobre el desarrollo de la inteligencia y su relación con el despliegue de elementos musicales propuesta por Moog (1976).

Tabla IV. Interpolación de las teorías del pensamiento de Piaget, Vygotsky y Moog

\begin{tabular}{|c|c|c|c|c|c|}
\hline $\begin{array}{l}\text { Etapas de } \\
\text { Piaget }\end{array}$ & $\begin{array}{l}\text { Sensomotora } \\
0 \text { a } 2 \text { años }\end{array}$ & $\begin{array}{l}\text { Pre-op } \\
2 \mathrm{a}\end{array}$ & $\begin{array}{l}\text { eracional } \\
5 \text { años }\end{array}$ & $\begin{array}{l}\text { Operaciones } \\
\text { concretas } \\
7 \text { a } 10 \text { años }\end{array}$ & $\begin{array}{l}\text { Operaciones formales } \\
\text { A partir de los } 11 \text { años }\end{array}$ \\
\hline $\begin{array}{l}\text { Desarrollo } \\
\text { del símbolo }\end{array}$ & \multicolumn{5}{|c|}{ mitación } \\
\hline $\begin{array}{l}\text { Desarrollo } \\
\text { de la noción } \\
\text { del tiempo }\end{array}$ & \multicolumn{5}{|c|}{$\begin{array}{l}\text { No consigue construir un tiempo único } \rightarrow \text { Invierte la relación del tiempo y de la velocidad } \rightarrow \text { Poco a } \\
\text { poco llega a la consecución del tiempo }\end{array}$} \\
\hline $\begin{array}{l}\text { Etapas de } \\
\text { Moog }\end{array}$ & \multicolumn{5}{|c|}{$\begin{array}{c}\text { Canto espontáneo } \rightarrow \text { Desarrollo Rítmico } \rightarrow \text { Imitación de canciones } \rightarrow \text { Desarrollo Melódico } \rightarrow \\
\text { Desarrollo Tonal }\end{array}$} \\
\hline $\begin{array}{l}\text { Etapas de } \\
\text { Vygotsky }\end{array}$ & Primitiva & $\begin{array}{l}\text { Psicología } \\
\text { simple }\end{array}$ & Egocéntrica & Crecimiento inte & \\
\hline
\end{tabular}

De acuerdo con esta interpolación, hay correlación en los estadios entre la formación de las habilidades cognitivas presentadas y el desarrollo del pensamiento musical.

Por otra parte, una de las críticas más fuertes a las teorías piagetianas ha sido que, a diferencia de los autores del constructivismo social, no consideran el aspecto sociocultural para el desarrollo de sus conceptos (Santoianni y Striano, 2006). Al respecto, es necesario hacer las siguientes aclaraciones:

1) En el marco de la presente investigación consideramos que Piaget sí tomó en cuenta el aspecto sociocultural para el desarrollo de sus teorías. Sin embargo, profundizó en el desarrollo cognitivo del niño desde un aspecto más individual.

2) Dada la razón anterior, las teorías de Piaget y Vygotsky son complementarias, pues es tan importante tomar en cuenta los aspectos individuales como los psicosociales, a fin de tener una comprensión completa del fenómeno. ${ }^{3}$

\subsection{Pautas para la implementación de metodología para la enseñanza musical}

El contexto. Para la selección de la metodología adecuada es necesario el análisis inicial del contexto, pues la enseñanza musical se desarrolla en varios ámbitos y cada uno de ellos presenta sus propios problemas. Por ejemplo, en la educación musical que se imparte en la escuela primaria se ha detectado en algunos países que, a falta de profesorado debidamente capacitado, la enseñanza de música se suprime. Algunas instituciones encuentran dificultades para tener instrumentos e instalaciones adecuadas. Además, las escuelas profesionales de música que se crearon para combatir dicha carencia, no se dan abasto. Las opciones son ingresar a una escuela privada que ofrezca clases de música o las clases particulares, pero no todas las personas tienen los recursos. En ese caso, los profesores o interesados en aprender pueden encontrar algunas opciones con métodos autodidactas o recursos en Internet como tutoriales y cursos en línea. En el caso de la falta de instrumentos musicales, se puede emplear el uso de

\footnotetext{
3 Hay un texto en el que Piaget responde a las críticas realizadas por Vygotsky (Piaget, 1962).
} 
la voz o instrumentos de percusión adaptados con diversos utensilios caseros. ${ }^{4}$

Las edades y habilidades de los participantes. También este elemento es fundamental para la selección de los materiales didácticos, dado que las edades de los participantes pueden oscilar es importante tomar en cuenta que los procesos cognitivos son distintos para diseñar la estrategia didáctica y seleccionar el método adecuado. Además, para el caso de las clases grupales, se debe considerar una forma de homogeneizar el ambiente. También se debe saber si los candidatos cuentan o no con habilidades musicales. Hay que tener presentes las cualidades físicas y mentales, si son de tipo promedio, si se trata de alumnado con capacidades distintas, etc. En el caso de grupos con características distintas puede funcionar una división interna a fin de que los más hábiles lleven ejercicios acordes a ellos. Habría que recurrir a métodos desarrollados para cada sector: bebés, niños, adolescentes, etc.

Elementos pedagógicos aplicables a los métodos de enseñanza musical. Se consideran los siguientes principios para la implementación de métodos de enseñanza:

Despliegue gradual y progresivo de contenidos. Es deseable que el desarrollo o adaptación de metodologías de enseñanza en una etapa inicial sigan las etapas formativas de la percepción musical: ritmo, melodía y armonía de forma gradual (Moog, 1976).

Tocar música cercana a los educandos. Una de la formas de adaptación del concepto ausubeliano de aprendizaje significativo es por medio de la implementación de elementos con los cuales los participantes estén previamente relacionados, incluso se pueden usar melodías o piezas sugeridas por los propios participantes. Así, el uso de melodías populares entre los educandos, relaciones algebraicas simples y la aplicación de colores puede favorecer la asimilación de nuevos conceptos.

Didáctica práctica $\rightarrow$ teoría $\rightarrow$ práctica. Los textos para la enseñanza musical están constituidos por una serie de fragmentos musicales que, además de desarrollar la musculatura del aprendiz, sirven para ilustrar conceptos propios del lenguaje musical. Para que el alumnado los realice de una manera más sencilla puede ser de mucha utilidad que los docentes hagan los ejercicios primero, el alumno los imite, posteriormente se enseñe cómo se representan mediante la escritura musical y por último se lean del pentagrama. En ese sentido, la didáctica recomendada puede desplegarse a través del proceso práctica/imitación $\rightarrow$ teoría/observación/análisis $\rightarrow$ práctica/sincretismo.

Desarrollo de modelos grupales para la enseñanza instrumental. Todavía prevalece la creencia de que la clase de música académica debe de ser individual, lo que hace muy costoso este tipo de modelos. Sin embargo, por medio del aprendizaje social, la conformación de grupos de enseñanza musical puede ayudar al proceso de aprendizaje a través de la experiencia de los individuos más desarrollados. La inclusión de tutores puede apoyar el proceso de enseñanza de los educandos, especialmente cuando son los padres los asesores de niños pequeños. Para ello, el docente encargado debe fomentar la interacción musical de los participantes, por medio de la implementación de piezas de cámara, o ponerles la misma pieza para que ellos intercambien sus experiencias.

Música de cámara con los estudiantes. Esta metodología tiene la ventaja de que el educando cuenta con la guía rítmica y armónica del profesor. Además, formar parte de un conjunto puede producir un resultado musical más interesante, lo que puede motivar al alumnado, sobre todo al inicio del proceso de enseñanza. En la figura 3 se presenta una propuesta para el desarrollo o adaptación de textos de enseñanza musical.

4 En México existen proyectos como el de Orquesta basura que interpreta su música a partir de instrumentos informales construidos con basura y artefactos de uso cotidiano. Para más información el lector puede consultar el sitio http://www.orquestabasura.com 


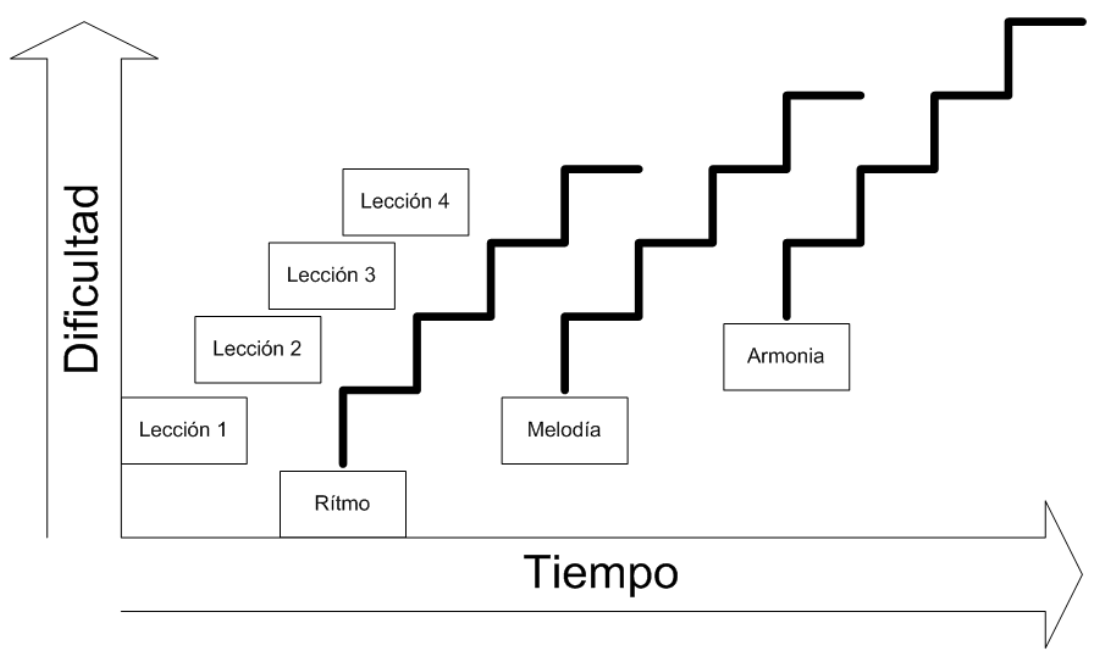

Figura 3. Esquema sobre el desarrollo de materiales didácticos musicales

\subsection{Condiciones para el uso de tecnología educativa y el diseño de plataformas en línea}

El primer paso es revisar una amplia gama de recursos tecnológicos aplicables a procesos de enseñanza, esta revisión debe hacerse de forma periódica, ya que la actualización, obsolescencia y aparición de herramientas es constante, luego hay que realizar un análisis para conocer sus posibilidades y seleccionar herramientas acordes al contexto teniendo las siguientes consideraciones:

Los espacios hipermedia facilitan el proceso de comunicación. Se ha comprobado que el desarrollo tecnológico permite la creación de sitios donde texto, audio, video y animación pueden amalgamarse para construir una representación simbólica del mundo, esto es llamado "espacios hipermedia" (Webster, 2002). Así, es posible generar ambientes para que los educandos puedan imitar los ejercicios e implementar esta práctica como medio didáctico en clase complementando la didáctica práctica-teoríapráctica. También la inclusión de mapas conceptuales puede compactar la información más relevante. Entonces, con el diseño de plataformas potenciadas con espacios hipermedia se pueden posibilitar espacios de práctica para que los estudiantes repasen los ejercicios y conceptos desde sus casas.

Incluir herramientas accesibles a los participantes. Es recomendable la incursión de tantas herramientas tecnológicas como sea posible, siempre y cuando apoyen el proceso de enseñanza de la interpretación musical. Pero es importante que cada uno de dichos recursos no complique el proceso de aprendizaje. Además, se sugiere que la navegación de los niños sea supervisada por un adulto.

Diseño de modelos didácticos. El diseño que el docente elija debe tomar en cuenta las distintas modalidades: presencial, en línea o híbrida. En ese sentido, Moodle, Blogger o Google Sites son plataformas que ofrecen toda una gama de posibilidades tecnológicas aplicables para la enseñanza musical.

En la figura 4 se observa una propuesta de espacio como facilitadora del proceso de comunicación, en esta propuesta hay dos tipos de interacción: una tecnológica a través de la interfaz entre el estudiante y la plataforma, y otra social en el aula, entre el estudiante y el profesor y entre el estudiante y otros estudiantes. 


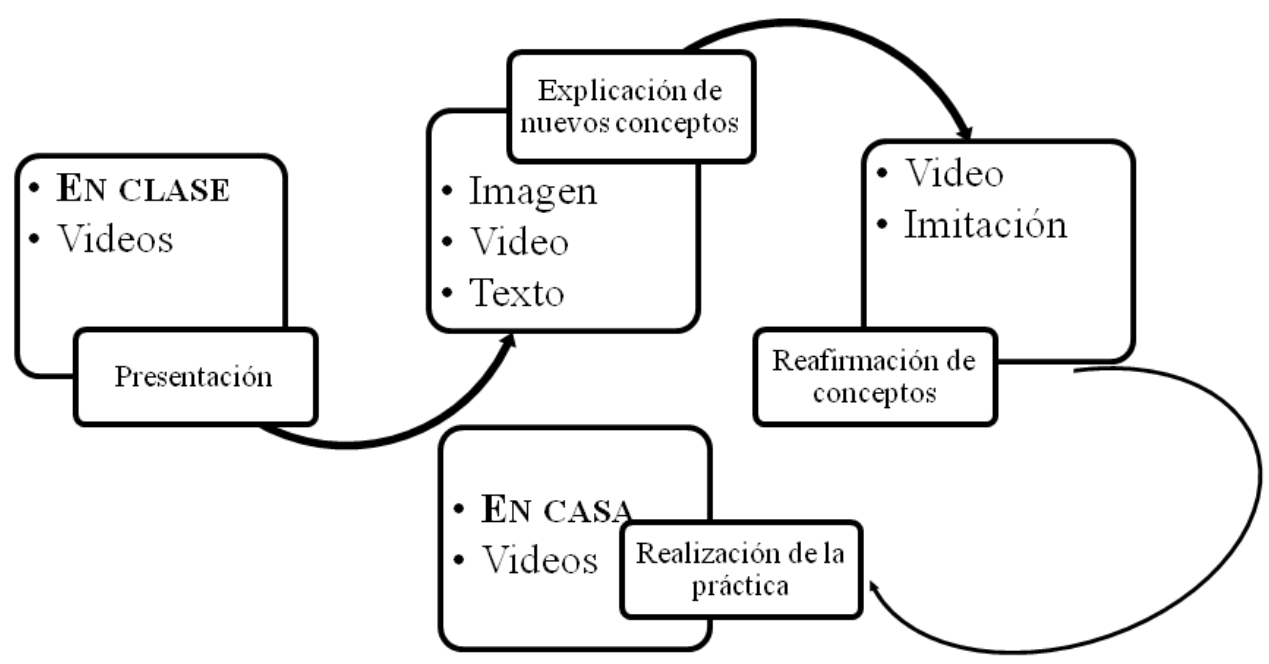

Figura 4. Esquema de un curso para guitarra en línea

\section{Conclusiones}

Es posible que el lector especialista de este trabajo eche de menos resultados que palpen el impacto en la puesta en práctica del decálogo didáctico expuesto; sin embargo, el objetivo de este artículo no es presentarlos. ${ }^{5}$ En cambio, enlistamos aquí las siguientes pautas para la implementación y el desarrollo de métodos de enseñanza musical:

Considerar el contexto para la selección de la o las metodologías más adecuadas. Esta selección debe incluir además de las estrategias didácticas, el instrumental que se utilizará, así como los contenidos. Para ello se deben tomar en cuenta:

- Edad de los participantes

- Habilidades del alumnado

- Gustos musicales de los sujetos

- Situación socioeconómica de los padres

Principios para el desarrollo de métodos de enseñanza:

- Despliegue gradual y progresivo de contenidos

- Incluir música cercana a los educandos

- Desarrollar una didáctica iniciada por la práctica-imitación, seguida por la revisión de teoríaobservación-análisis, para cerrar las sesiones con la práctica-sincretismo de los conceptos

- Tocar música junto con los estudiantes

El problema con la enseñanza de la música abarca muchos aspectos acordes al contexto de que se trate. Por esta razón, la eficacia de un método de enseñanza puede ser muy alta o discreta, si el lugar en el que se aplica no es el adecuado. Así, el músico que se va a iniciar como profesor debe documentarse y realizar un diagnóstico preliminar para determinar las cualidades del estudiantado y seleccionar el método más adecuado. Podemos determinar que el método ideal no existe, en cambio tenemos una amplia gama de modelos, textos y metodologías de enseñanza que bien pueden implementarse o adaptarse en diversos contextos. Para ello es indispensable que los docentes realicen una extensa exploración sobre los modelos y textos didácticos para poder implementar el más adecuado. Hoy con el surgimiento de las

5 El lector puede acceder a artículos de investigación que muestran resultados de procesos de investigación realizados a partir de cursos de música implementados bajo las pautas que aquí presentamos. Consultar Navarro, Lavigne y Martínez (2009), y Navarro, Lavigne y Tejada (2013). 
Tecnologías del Aprendizaje y el Conocimiento (TAC), ${ }^{6}$ se demuestra que el simple conocimiento de herramientas tecnopedagógicas no es suficiente para que haya una incidencia sustancial en los procesos de enseñanza y aprendizaje de las sociedades contemporáneas. De la misma manera, el simple conocimiento de métodos de enseñanza musical no mejorará los procesos educativos.

\section{Referencias}

Alsina, P. (2007). Métodos de enseñanza musical: algunos puntos de contacto. En M. Díaz y A. Giráldez (Coords.), Aportaciones teóricas y metodológicas a la educación musical: una selección de autores relevantes. Barcelona: Graó.

Ausubel, D., Novak, J. y Hanesian, H. (2006). Psicología Educativa. Un punto de vista cognoscitivo. México: Trillas.

Bunge, M. (1980). Ciencia y desarrollo. Buenos Aires: Siglo XX.

Burgos, A. (2007). Los métodos de enseñanza en la educación musical. Recuperado de http://weblog.educ.ar/espacio docente/musica/archives/001745.php

Enríquez, S. (2012). La formación básica para docentes y las TAC. Educación y TIC. Blog de la Dirección de Educación a Distancia de la Universidad Nacional de La Plata. Recuperado de http://www.ead.unlp.edu.ar/blog/?p=209

Ferreiro, R. (2007). Una visión de conjunto a una de las alternativas educativas más impactante de los últimos años: el aprendizaje cooperativo. Revista Electrónica de Investigación Educativa, 9(2). Recuperado de http://redie.uabc.mx/redie/article/view/176

Furnó, S. (2005). La formación de conceptos musicales. Cuadernos Interamericanos de Investigación en Educación Musical, 2(6), 81-108.

Gardner, H. (2005). Inteligencias múltiples: la teoría en la práctica. Barcelona: Paidós.

Hernández, G. (2010). Paradigmas en psicología de la educación. México: Paidós.

Jorquera, M. (2004). Métodos históricos o activos en educación musical. Lista Europea de Música en la Educación, 14. Recuperado de http://musica.rediris.es/leeme/revista/jorquera04.pdf

Joyce, B., Weil, M. y Calhoun, E. (2006). Modelos de enseñanza. Barcelona: Gedisa.

Lara, T. (2005). Blogs para educar: Usos de los blogs en una pedagogía constructivista. Telos, 2. Recuperado de http://www.campusred.net/telos/articulocuaderno.asp?idarticulo=2\&rev=65

Moog, H. (1976). The musical experience of the pre-school child. Londres: Schott.

Muñoz, J. (2003). El juego en la educación musical. Jugar con la música. Eufonía 29. Barcelona: Graó.

Navarro, J. L., Lavigne, G. y Martínez, G. (2009). Curso de guitarra clásica en línea: blogs para la enseñanza musical. Lista Europea Electrónica de Música en la Educación, 24. Recuperado de http://musica.rediris.es/leeme/revista/navarroetal09.pdf

\footnotetext{
6 Las TAC asegurarán que los docentes puedan incorporar a sus clases las nuevas posibilidades que ofrece la educación a distancia (Enríquez, 2012).
} 
Navarro, J. L., Lavigne, G. y Tejada, J. (2013). Un modelo distribuido de curso en línea de guitarra clásica. Lista Europea Electrónica de Música en la Educación, 32. Recuperado de http://musica.rediris.es/leeme/revista/navarroetal13.pdf

Piaget, G. (1996). Estudios de psicología genética. Buenos Aires: Emecé.

Piaget, G. (2005). El desarrollo de la noción de tiempo en el niño. México: Fondo de Cultura Económica.

Piaget, G. (2006). La formación del símbolo en el niño. México: Fondo de Cultura Económica.

Piaget, J. (1962). Comments on Vygotsky's critical remarks concerning The Language and Thought of the Child, and Judgment and Reasoning in the Child. Recuperado de

http://www.marxists.org/archive/vygotsky/works/comment/piaget.htm

Piñeiro, E. (1986). Para una Metodología de la Enseñanza de la Guitarra. La Habana: Pueblo y Educación.

Santoianni, F. y Striano, M. (2006). Modelos teóricos y metodológicos de la enseñanza. México: Siglo XXI.

Sierra, F. (2002). Las computadoras en la escuela. Ciderhábitat Ciudad de la Informática. Recuperado de http://ciderhabitat.gob.mx/escuela/maestro

Vygotsky, L. (1979). El desarrollo de las funciones psicológicas superiores. Barcelona: Grijalbo.

Vygotsky, L. (2008). Pensamiento y Lenguaje. México: Quinto Sol.

Webster, P. (2002). Historical Perspectives on technology and Music. Music Educators Journal, 89(1), 3843.

Wikipedia Org. (2014). Wikipedia Enciclopedia. Recuperado de http://es.wikipedia.org/wiki/Wikipedia:Portada 